\title{
Deutschsprachige Terminologie der revidierten Atlanta- Klassifikation bei akuter Pankreatitis: Glossar basierend auf der aktuellen S3-Leitlinie zur akuten, chronischen und Autoimmunpankreatitis
}

\section{German Terminology of the Revised Atlanta Classification of Acute Pancreatitis: Glossary Based on the New German S3 Guideline on Acute, Chronic, and Autoimmune Pancreatitis}

\section{Autoren}

Andreas G. Schreyer ${ }^{1}$, Max Seidensticker ${ }^{2}$, Julia Mayerle ${ }^{3}$, Markus M. Lerch ${ }^{4}$, Markus S. Juchems ${ }^{5}$, Johannes Wessling ${ }^{6}$, Kristina Imeen Ringe ${ }^{7}$, Lars Grenacher ${ }^{8}$

Institute

1 Institute for Diagnostic and Interventional Radiology, Brandenburg Medical School Theodor Fontane, Brandenburg a.d. Havel, Germany

2 Dpt. of Radiology, University Hospital, LMU Munich, Munich, Germany

3 Department of Medicine II, LMU Munich, Munich, Germany

4 Department of Medicine A, University Medicine Greifswald, Greifswald, Germany

5 Diagnostic and Interventional Radiology, Hospital Konstanz, Germany

6 Department of Radiology, Clemenshospital GmbH Münster, Germany

7 Department of Diagnostic and Interventional Radiology, Hannover Medical School, Hannover, Germany

8 Imaging and Prevention Center, Conradia Radiology Munich, Germany

\section{Key words}

acute pancreatitis, revised Atlanta classification, guideline, glossary, german translation

eingereicht 10.12 .2020

akzeptiert 23.01.2021

online publiziert 18.03.2021

\section{Bibliografie}

Fortschr Röntgenstr 2021; 193: 909-918

DOI 10.1055/a-1388-8316

ISSN 1438-9029

(C) 2021. Thieme. All rights reserved.

Georg Thieme Verlag KG, Rüdigerstraße 14,

70469 Stuttgart, Germany
Korrespondenzadresse

Prof. Lars Grenacher

Imaging and Prevention Center, Conradia Radiology Munich,

Augustenstr. 115, 80798 München, Germany

Tel.: $+49 / 89 / 52055200$

Fax: $+49 / 89 / 52055203$

l.grenacher@diagnostik-muenchen.de

\section{ZUSAMMENFASSUNG}

Die im Jahr 1992 veröffentlichte Atlanta-Klassifikation zur Beurteilung von Schweregraden und Komplikationen der akuten Pankreatitis sollte ursprünglich eine einheitliche Terminologie für Kliniker und Forscher etablieren. Nach einer kontinuierlichen Weiterentwicklung wurde dann 2012 die revidierte Atlanta-Klassifikation publiziert, um die Aspekte der Bildgebung besser zu integrieren sowie Unschärfen in der Terminologie zu optimieren. Diese Begrifflichkeiten bezüglich der Krankheitseinteilung, -phasen, Schweregradbeurteilung und lokaler Komplikationen lagen bisher lediglich als englische Ausdrücke vor. Dies verhinderte eine weitere Verbreitung und Anwendung im klinischen Alltag im deutschsprachigen Raum. Um eine Anpassung in der deutschsprachigen Medizin zu erreichen, wurden die Termini der revidierten Atlanta-Klassifikation durch ein Expertenteam der Deutschen Röntgengesellschaft basierend auf aktueller Literatur übersetzt und im Rahmen der Konsensuskonferenz der neu erstellten S3-Leitlinie Pankreatitis im Februar 2020 mit einer Zustimmung von 97,3\% als Teil der Leitlinienempfehlung angenommen. Im folgenden Text mit Glossar werden die übersetzten und konsentierten Begrifflichkeiten anhand von Bildbeispielen erläutert.

\section{Kernaussagen:}

- Die revidierte Atlanta-Klassifikation ermöglicht eine korrekt definierte Bezeichnung von Krankheitsstadien und Komplikationen der akuten Pankreatitis.

- Die von einer Expertengruppe ins Deutsche übersetzten Begrifflichkeiten wurden im Rahmen der neuen S3-Leitlinie Pankreatitis konsentiert.

- Basierend auf der neuen S3-Leitlinie Pankreatitis sollen die deutschen Begriffe konsequent verwendet werden. 


\section{ABSTRACT}

The Atlanta classification published in 1992 was supposed to classify the severity and complications in acute pancreatitis with the goal of providing uniform terminology for clinicians and researchers. After continuous evolution, the revised Atlanta classification was published in 2012. In this updated classification, imaging aspects were better integrated and unclear terms were better defined. To date, this terminology for disease grading, severity, and local complications has been solely available in English. This prevented wide acceptance of the classification by the German-speaking medical community. Therefore, an expert team of the German Radiological Society translated the terms based on German medical literature. This translation was approved by consensus in the new $\mathrm{S3}$ guideline meeting in February 2020 with an approval rate of $97.3 \%$. This manuscript explains the translated and approved German terminology of the revised Atlanta classification and offers additional imaging examples.

\section{Key Points:}

- The revised Atlanta classification allows correct definition of the grading and complication assessment of acute pancreatitis.

- An expert group translated the terms into the German language. The translation was approved by the $\mathrm{S} 3$ guideline committee for pancreatitis.

- Based on the new German S3 guideline for pancreatitis, the translated terms should be employed consistently.

\section{Citation Format}

- Schreyer AG, Seidensticker M, Mayerle J et al. German Terminology of the Revised Atlanta Classification of Acute Pancreatitis: Glossary Based on the New German S3 Guideline on Acute, Chronic, and Autoimmune Pancreatitis. Fortschr Röntgenstr 2021; 193: 909-918

\section{Einleitung}

Die im Jahr 1992 etablierte Atlanta-Klassifikation hatte zum Ziel, interdisziplinär und Konsensus-basiert eine einheitliche Terminologie für verschiedene Schweregrade der akuten Pankreatitis (AP) zu definieren [1]. Zusätzlich wurden Begrifflichkeiten für Komplikationen einer akuten Pankreatitis im Rahmen der Klassifikation veröffentlicht, die eine strukturierte und klare Kommunikation zwischen den an der Diagnose und Behandlung der AP beteiligten Disziplinen erlauben soll.

Die Atlanta-Klassifikation wurde damals von Vertretern aus Gastroenterologie, Radiologie und Chirurgie zusammen mit weiteren Disziplinen während eines 3-tägigen Treffens zusammengestellt. Ziel war es, eine klinisch basierte Klassifikation für die AP zu schaffen, die nicht nur von Radiologen, sondern vor allem auch von den patientenführenden Disziplinen, aber auch Forschern, als gemeinsame Sprache und Terminologie der AP benutzt werden kann.

In der ursprünglichen Version von 1992 erfolgte lediglich eine Einteilung in eine sogenannte leichte Pankreatitis (mild pancreatitis) und eine schwere Pankreatitis (severe pancreatitis). Bei den Komplikationen wurde zwischen einer „acute fluid collection“, einer akuten Pseudozyste sowie Pankreasabszess und Pankreasnekrose unterschieden.

Kritisch gesehen wird bei dieser ursprünglichen Version, dass sie sich vor allem auf klinische Scores (RANSON oder APACHE II) bezieht. Zusätzlich existiert keine adäquate Korrelation der klinischen Präsentation, des Schweregrades und der radiologischen Kriterien, die lokale Komplikationen beschreiben [2]. Gerade bei den assoziierten Komplikationen gibt es keine adäquaten radiologischen Definitionen, besonders im Hinblick auf Flüssigkeitsansammlungen bei der AP, die als akute Pseudozysten (acute pseudocyst) oder als Pankreasabszess (pancreatic abscess) zu einer uneinheitlichen und damit klinisch verwirrenden Begriffsverwendung führten. Darüber hinaus gab es keine strukturierten morphologischen Ausdrücke, beispielsweise zur Definition einer peripankreatischen oder einer pankreatischen Nekrose.
Im Laufe der Jahre wurde den mit der Pankreatitis vertrauten Experten zunehmend bewusst, dass eine Revision dieser Klassifikation nötig war, um die Fortschritte in der Bildgebung besser zu integrieren, aber auch die inkonsequente Benutzung der Terminologie zu optimieren. Kritisch wurde auch gesehen, dass die in der Atlanta-Klassifikation von 1992 verwendeten Begriffe klinisch teilweise sehr heterogen und ungenau definiert wurden [3]. 20 Jahre nach Etablierung der ursprünglichen Klassifikation erfolgte dann im Jahr 2012 die Verabschiedung der sogenannten revidierten Atlanta-Klassifikation [4]. Diese revidierte Version hatte zum Ziel, eine klar definierte gemeinsame Terminologie zu schaffen, um unter anderem den Schweregrad der Pankreatitis, aber auch die lokalen Komplikationen wie etwa Flüssigkeitsansammlungen korrekt nach Erkrankungsdauer und Form bildgebend eindeutig zu beschreiben.

\section{Diagnose}

In der revidierten Atlanta-Klassifikation wird die Diagnose einer AP gestellt, wenn mindestens 2 der folgenden 3 Kriterien zutreffen: (1) charakteristische typische (gürtelförmige) Bauchschmerzen, (2) Erhöhung der Serumlipase und Amylase um mehr als Faktor 3 des maximalen Normwertes sowie (3) bildgebender Nachweis von Veränderungen des Pankreas.

\section{Einteilung}

Bezüglich der physiologisch-anatomischen Einteilung der AP unterscheidet die revidierte Klassifikation die AP in eine interstitielle ödematöse Pankreatitis (ca. 85 \%) und eine nekrotisierende Pankreatitis (15\%). Die Nekrosen werden wiederum topografisch in eine Nekrose des Pankreasparenchyms (ca. $5 \%$ ), des peripankreatischen Gewebes (ca. $20 \%$ ) sowie in eine Kombination (75\%) der beiden unterteilt. 


\section{Phasen}

Klinisch wird der Verlauf der AP in eine Frühphase (early phase) und eine Spätphase (late phase) unterteilt. In der Frühphase liegt in der Regel eine systemische Inflammation vor. Dabei wird die Frühphase als Veränderungen innerhalb der ersten Woche nach dem ersten Auftreten der Symptome definiert. Die klinische Spätphase beschreibt im Anschluss Veränderungen ab der 2. Woche. Die Bildgebung ist bei fehlendem Hinweis auf Komplikationen in der Regel erst in der Spätphase ab der 2. Woche sinnvoll, frühestens jedoch nach 72 Stunden, da sich vorher eventuelle Nekrosen im Parenchym noch nicht demarkiert haben ( $\triangleright$ Abb. 1a, b).

\section{Schweregradbeurteilung}

Der Schweregrad der AP wird prinzipiell basierend auf möglichem Organversagen sowie lokalen und systemischen Komplikationen unterteilt. Im englischen Originaltext wird die Pankreatitis als „mild“ bezeichnet, wenn kein Organversagen und keine Organkomplikation vorliegen. Bei einem transienten Organversagen (Dauer < 48 Stunden) mit fakultativem Vorliegen von lokalen Komplikationen wird die AP als "moderatly severe" bezeichnet. Die sogenannte „severe“ AP liegt bei Organversagen von mehr als 48 Stunden sowie bei lokalen und systemischen Komplikationen vor und weist Mortalitätsraten von 20-30\% auf.

\section{Lokale Komplikation}

Die ursprüngliche Atlanta-Klassifikation von 1992 definierte lokale Komplikationen mit Begriffen wie „acute fluid collections“, „acute pseudocyst“ sowie „pancreatic abscess“. Ein Problem mit diesen Bezeichnungen lag in der fehlenden scharfen Trennbarkeit und Definition mit konsekutiv schlechten Intra-Observer-Übereinstimmungen.

In der revidierten Atlanta-Klassifikation werden die lokalen Komplikationen vor allem durch die Bildgebung, insbesondere in Form der kontrastgestützten Computertomografie, definiert. Prinzipiell erfolgt eine Einteilung der lokalen Komplikationen basierend auf dem Zeitpunkt nach Auftreten der Erkrankung sowie der Einteilung in die interstitiell-ödematöse und in die nekrotisierende Pankreatitis ( $\triangleright$ Tab. 1). Als „acute“ bei lokalen Komplikationen wird der Zeitraum innerhalb der ersten 4 Wochen nach Auftreten der Erkrankung definiert, als „delayed“ bei lokalen Komplikationen nach 4 Wochen. Diese Begrifflichkeiten sollten nicht mit den Phasen der Erkrankung verwechselt werden, die als „early phase“ und "late phase“ (siehe oben $\rightarrow$ Phasen) definiert sind.

\section{Verwendung der Terminologie und Übersetzung}

Auch wenn die Atlanta-Klassifikation in ihrer revidierten Fassung seit 2012 existiert, wird sie dennoch gegenwärtig nicht konsequent im deutschsprachigen Raum eingesetzt. Obgleich die Klassifikation publiziert wurde, um eine einheitliche Sprache in Klinik und Wissenschaft interdisziplinär zu schaffen, verhindert die feh-
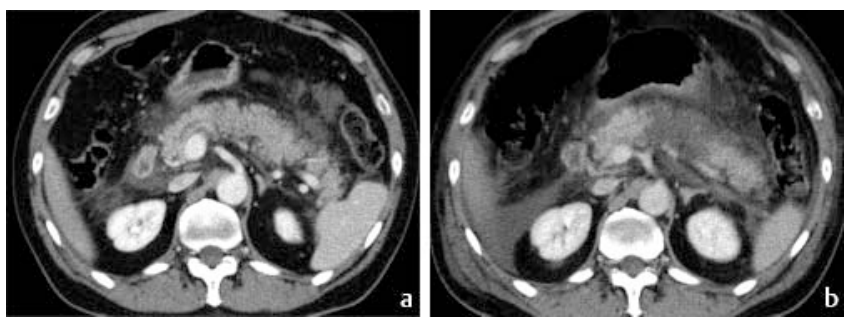

- Abb. 1 a akute Pankreatitis mit peripankreatischer freier Flüssigkeit innerhalb 72 Stunden. b erst nach 72 Stunden demarkiert sich die akute Nekrose (ANC) im Pankreaskorpus.

lende Anpassung und Übersetzung in andere Sprachen außerhalb des angloamerikanischen Sprachraums sicherlich eine Verbreitung und Akzeptanz in der täglichen Routine.

Um die Grundlage für eine bessere Akzeptanz der revidierten Atlanta-Klassifikation für Patienten mit AP zu erreichen, wurde zunächst von Experten im Vorstand der Arbeitsgemeinschaft Abdominal- und Gastrointestinaldiagnostik in der Deutschen Röntgengesellschaft (DRG) der Versuch unternommen, basierend auf Literaturrecherche und deutschsprachigen Publikationen eine adäquate Übersetzung der Begrifflichkeiten der revidierten Atlanta-Klassifikation für den deutschsprachigen Raum zu schaffen.

Um eine möglichst globale und rasche Akzeptanz dieser Terminologie in Deutschland zu erreichen, wurde diese übersetzte Terminologie im Rahmen der Konsensuskonferenz der S3-Leitlinie akute und chronische Pankreatitis vom 11.-13. Februar 2020 in Halle/Saale den interdisziplinären Experten der AWMF-Leitlinie zur Abstimmung vorgelegt, weiterentwickelt und diskutiert.

Im Rahmen der Leitlinienkonferenz und Abstimmung am 12. Februar 2020 erhielt der Übersetzungsvorschlag für eine vereinheitlichte deutsche Terminologie der revidierten Atlanta-Klassifikation eine Zustimmung von 97,3\% unter den interdisziplinären Experten der Leitlinienteilnehmer, was einen starken Konsens zur Annahme belegt.

In der Leitliniengruppe einigte man sich darauf, künftig im deutschsprachigen Raum die übersetzten Begriffe benutzen zu wollen. Anders als im englischsprachigen Original sollten die ins Deutsche übersetzten Begriffe jedoch nicht durch deutsche Abkürzungen der Begriffe ergänzt werden, sondern ggf. mit der englischen Originalabkürzung. So sollte beispielsweise die Spätkomplikation einer nekrotisierenden akuten Pankreatitis, die durch eine abgekapselte Flüssigkeitsansammlung definiert ist und im englischsprachigen Original als „walled off necrosis“ (WON) bezeichnet wird, im deutschen Text als abgekapselte Nekrose beschrieben werden, und dabei gleichzeitig mit der englischen Abkürzung WON bezeichnet werden (siehe $>$ Tab. 1).

Im Folgenden sollen die Begriffe aus der revidierten AtlantaKlassifikation im englischen Original thematisch geordnet mit ihrer deutschen Übersetzung erörtert und definiert und anhand von typischen radiologischen Bildern als illustriertes Glossar dargestellt werden. 
- Tab. 1 Abkürzungen: Acute Pancreatitis (AP). Die deutschen Begriffe sollten nicht durch eine deutsche Abkürzung, sondern durch die ggf. vorhandene englische Abkürzung (Spalte 2) ergänzt werden.

\begin{tabular}{|c|c|c|c|}
\hline Begriff (Original) & Abkürzung & Definition & deutscher Begriff \\
\hline Onset & & Beginn der abdominellen Schmerzen & Erkrankungsbeginn \\
\hline Early phase & & $<1$ Woche nach Erkrankungsbeginn & Frühphase \\
\hline Late phase & & $>1$ Woche nach Erkrankungsbeginn & Spätphase \\
\hline Acute complication & & $\begin{array}{l}<4 \text { Wochen nach Erkrankungsbeginn } \\
\text { (APFC, ANC) }\end{array}$ & frühe Komplikation \\
\hline Delayed complication & & $\begin{array}{l}\text { > } 4 \text { Wochen nach Erkrankungsbeginn } \\
\text { (WON, pancreatic pseudocyst) }\end{array}$ & späte Komplikation \\
\hline Mild AP & & kein Organversagen & leichte akute Pankreatitis \\
\hline Moderately severe AP & & $\begin{array}{l}\text { transientes Organversagen }(<48 \mathrm{~h}) \text { und/ } \\
\text { oder lokale Komplikationen }\end{array}$ & mittelschwere akute Pankreatitis \\
\hline Severe AP & & $\begin{array}{l}\text { persistierendes Organversagen ( }>48 \mathrm{~h} \text { ), oft } \\
\text { lokale und systemische Komplikationen }\end{array}$ & schwere akute Pankreatitis \\
\hline Collection & & $\begin{array}{l}\text { Ansammlung von Flüssigkeit und } \\
\text { nekrotischem Gewebe }\end{array}$ & Ansammlung \\
\hline Fluid collection & & Ansammlung von Flüssigkeit & Flüssigkeitsansammlung \\
\hline Interstitial edematous AP & & & interstitielle ödematöse Pankreatitis \\
\hline Necrotizing AP & & & nekrotisierende Pankreatitis \\
\hline $\begin{array}{l}\text { Acute peripancreatic fluid } \\
\text { collection }\end{array}$ & APFC & $\begin{array}{l}\text { frühe Komplikation einer interstitiellen } \\
\text { ödematösen AP - homogene Flüssigkeits- } \\
\text { ansammlung ohne Nekrose oder definierte } \\
\text { Abkapselung }\end{array}$ & akute peripankreatische Flüssigkeitsansammlung \\
\hline Pancreatic pseudocyst & & $\begin{array}{l}\text { späte Komplikation einer interstitiellen } \\
\text { ödematösen AP, charakterisiert durch eine } \\
\text { definierte, abgekapselte Flüssigkeitsan- } \\
\text { sammlung ohne relevante Nekroseanteile }\end{array}$ & Pankreas-Pseudozyste \\
\hline Acute necrotic collection & ANC & $\begin{array}{l}\text { frühe Komplikation einer nekrotisierenden } \\
\text { AP -Flüssigkeitsansammlung und Nekrose } \\
\text { ohne Nachweis einer Abkapselung }\end{array}$ & akute Nekrose \\
\hline Walled-off necrosis & WON & $\begin{array}{l}\text { späte Komplikation einer nekrotisierenden } \\
\text { AP, charakterisiert durch eine abgekapsel- } \\
\text { te Flüssigkeitsansammlung und Nekrose } \\
\text { des Pankreasparenchyms oder des peri- } \\
\text { pankreatischen retroperitonealen Fetts } \\
\text { oder beides }\end{array}$ & abgekapselte Nekrose \\
\hline
\end{tabular}

\section{Chronologische Einteilung der akuten Pankreatitis sowie deren Komplikationen}

\section{Onset}

Der Onset wird im Deutschen mit dem Begriff „Erkrankungsbeginn übersetzt und ist dabei definiert als der Zeitpunkt des Beginns der akuten abdominellen Schmerzen und klinischen Symptomatik. Bei der klinischen Dokumentation darf der Begriff nicht mit der ersten Kontaktaufnahme mit einem Arzt bzw. Vorstellung in der Notaufnahme verwechselt werden.

Die Definition des Erkrankungsbeginns ist konsekutiv die Grundlage für die beiden folgenden Definitionen der Frühphase und Spätphase der Erkrankung, die jeweils unterschiedliche diagnostische und therapeutische Konsequenzen haben.

\section{Early phase}

Dieser Begriff wird mit „Frühphase“ übersetzt und ist definiert als der Zeitraum innerhalb der ersten Woche nach Erkrankungsbeginn (onset). In dieser ersten Woche manifestiert sich die Erkrankung als eine systemische Entzündungsantwort, wobei die Krankheitsschwere sowie die Behandlung vor allem auf der Basis und des Grades des Organversagens bestimmt sind.

\section{Late phase}

Der Begriff wird mit „Spätphase“ übersetzt und beschreibt die Erkrankung ab der 2. Woche nach dem Erkrankungsbeginn. Die Spätphase kann Wochen bis Monate andauern und tritt vor allem bei Patienten mit mittelschwerer oder schwerer akuter Pankreatitis auf. 

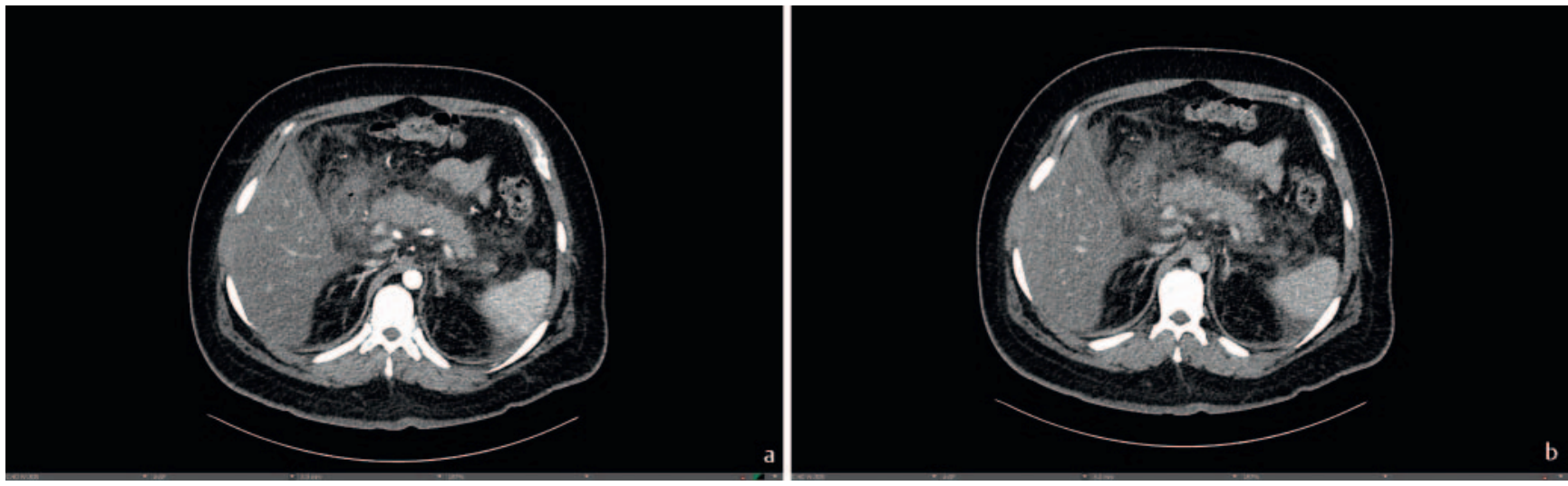

- Abb. 2 a Frühphase, interstitielle ödematöse Pankreatitis (APFC) mit Imbibierung des peripankreatischen Fettgewebes. b die peripankreatische Flüssigkeit reicht vom Pankreasschwanz entlang der Gerota Faszie bis um die Milz herum.

\section{Acute complication}

Dieser Terminus wird mit „frühe Komplikation“ übersetzt und beschreibt Komplikationen der AP, welche innerhalb der ersten 4 Wochen nach Erkrankungsbeginn zu finden sind. Beispielsweise ist bei der interstitiellen ödematösen akuten Pankreatitis die Frühkomplikation die akute peripankreatische Flüssigkeitsansammlung (APFC), bei der nekrotisierenden akuten Pankreatitis ist die typische Frühkomplikation eine akute Nekrose (ANC).

\section{Delayed complication}

Diese wird als „Spätkomplikation“ bezeichnet und ist definiert durch Komplikationen, die nach der 4. Woche nach Erkrankungsbeginn auftreten. Dabei ist z. B. die Pankreaspseudozyste die Spätkomplikation der interstitiellen ödematösen akuten Pankreatitis und die abgekapselte Nekrose (WON) die Spätkomplikation einer nekrotisierenden akuten Pankreatitis.

\section{Einteilung des Schweregrades der akuten Pankreatitis}

Um eine potenzielle Stratifikation der Patienten nach Schweregraden zu ermöglichen, wurde die Einteilung des Schweregrades in der revidierten Atlanta-Klassifikation geändert. Die ursprüngliche Klassifikation von 1992 teilte die Patienten entweder in eine schwere Pankreatitis (severe pancreatitis) oder eine leichte Pankreatitis (mild pancreatitis) auf der Basis der Anwesenheit oder Abwesenheit eines Organversagens ein. Es zeigte sich jedoch, dass ein Teil der Patienten mit lokalen Komplikationen und Flüssigkeitsansammlungen zwar eine beträchtliche Morbidität aufwiesen, jedoch nur eine geringe Mortalität hatten [2]. Daher wurde in der revidierten Atlanta-Klassifikation eine dritte Kategorie eingeführt, die „moderately severe acute pancreatitis“.

\section{Mild acute pancreatitis}

Der Begriff wird mit „leichte akute Pankreatitis“ im Deutschen übersetzt. Von einer unreflektierten direkten Übertragung des Begriffs „mild“ in den deutschen Begriff „mild“ sollte Abstand genommen werden. Es hat sich nämlich eingebürgert, den Begriff „mild“ auch in der deutschen Sprache zu verwenden (sog. „milde Pankreatitis“). Allerdings ist gemäß Duden das Adjektiv eher im Sinne von „gütig“ zu verstehen und daher nicht adäquat zur Beurteilung eines Schweregrades geeignet. Daher sollte hier der englische Begriff „mild“ (englisch) mit „leicht“ (deutsch) übersetzt werden. Bei der leichten AP tritt weder ein Organversagen noch eine lokale Komplikation auf. Bei diesen Patienten erfolgt zumeist eine stationäre Entlassung innerhalb der ersten Woche (Frühphase). Auch die Mortalität ist bei diesen Patienten äußerst gering [5].

\section{Moderately severe acute pancreatitis}

Dieser Schweregrad ist in der revidierten Atlanta-Klassifikation neu definiert und wird mit „mittelschwere akute Pankreatitis“ ins Deutsche übersetzt. Bei diesen Patienten tritt ein vorübergehendes Organversagen auf, das weniger als 48 Stunden dauert. Zusätzlich können ggf. lokale oder systemische Komplikationen auftreten. Häufig handelt es sich bei den systemischen Komplikationen um eine Exazerbation von bekannten Komorbiditäten, wie z. B. ein akutes Nierenversagen im Rahmen eines chronischen Nierenversagens. Die lokalen Komplikationen können ein breites Spektrum aufweisen: am häufigsten treten pankreatische bzw. peripankreatische Flüssigkeitsansammlungen auf. Diese Flüssigkeitsansammlungen entstehen meistens in der 2. Woche nach Krankheitsbeginn (Spätphase) und sind vor allem bei Patienten mit zunehmenden Schmerzen und Anstieg der pankreatischen Enzyme zu beobachten. Bei diesen Patienten sollte in dieser Phase die Bildgebung, am besten die kontrastgestützte Computertomografie oder auch kontrastgestützte MR-Tomografie, durchgeführt werden [6].

\section{Severe acute pancreatitis}

Der Terminus wird als „schwere akute Pankreatitis“ übersetzt. Bei diesen Patienten persistiert das Organversagen mehr als 48 Stunden mit regelmäßig vorhandenen systemischen oder lokalen Komplikationen. 

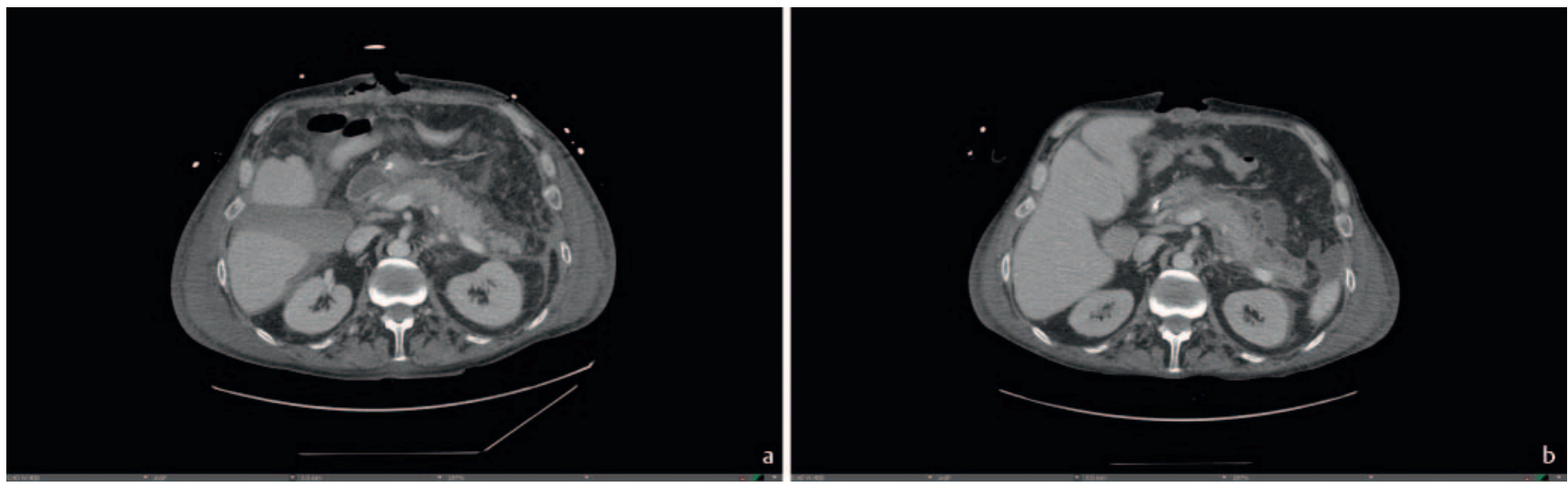

- Abb. 3 a Frühphase, interstitielle ödematöse Pankreatitis (APFC); b Verlauf nach 5 Wochen im Sinne einer späten Komplikation mit PankreasPseudozyste.

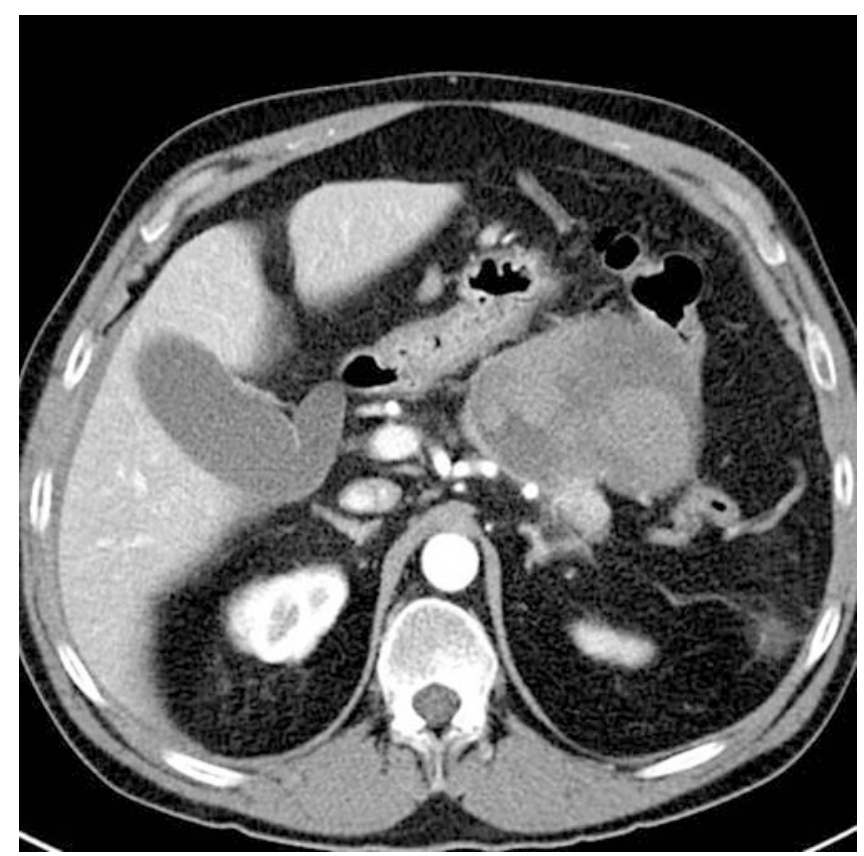

- Abb. 4 Große, eingeblutete Pseudozyste, typisch die Lokalisation in der Bursa omentalis bzw. peripankreatisch.

\section{Collection}

Der Begriff wird mit „Ansammlung“ übersetzt. Im Gegensatz zur Flüssigkeitsansammlung (fluid collection) besteht eine Ansammlung aus Flüssigkeit und gleichzeitig partiell nekrotischem Gewebe. In Zusammenhang mit lokalen Komplikationen wird dieser Begriff daher auch nur bei einer AP im Sinne einer nekrotisierenden Pankreatitis verwendet (acute necrotic collection).

\section{Fluid collection}

Der Begriff wird mit „Flüssigkeitsansammlung“ übersetzt und beschreibt die Ansammlung von Flüssigkeiten ohne nekrotisches Gewebe. Es wird daher als Komplikation einer interstitiell-ödematösen Pankreatitis bei der akuten peripankreatischen Flüssigkeitsansammlung (APFC) verwendet.

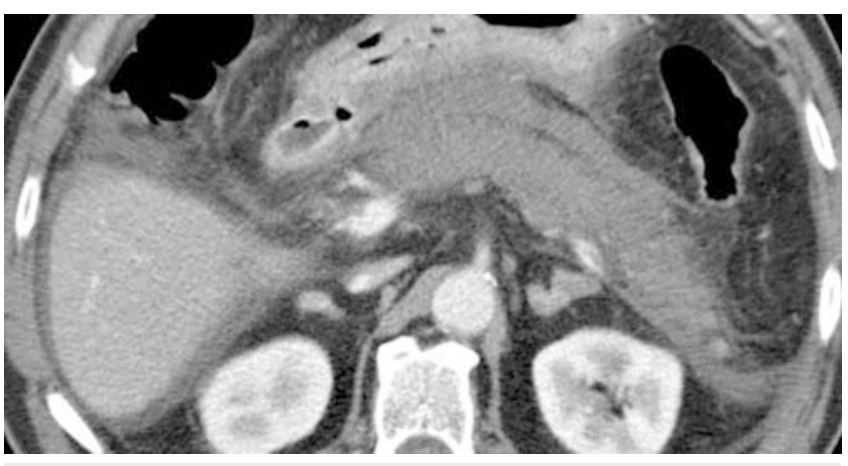

Abb. 5 Fast homogene Hypodensität (Perfusionsausfall) des Pankreas, keine KM-Aufnahme des Parenchyms, einer akuten Totalnekrose entsprechend.

\section{Interstitial edematous acute pancreatitis}

Prinzipiell wird in der revidierten Atlanta-Klassifikation die AP in eine „interstitiell-ödematöse Pankreatitis“ und in eine „nekrotisierende Pankreatitis“ eingeteilt.

Die interstitiell-ödematöse Pankreatitis ist mit 85 \% die prädominante Verlaufsform und korreliert in vielen Fällen mit einer leichten akuten Pankreatitis. Bei kontrastmittelgestützten Untersuchungen nimmt bei der interstitiell-ödematösen Pankreatitis das gesamte Pankreas Kontrastmittel auf und zeigt keinerlei Areale mit fehlender Anreicherung. Die interstitiell-ödematöse Pankreatitis zeigt sich am häufigsten mit fokaler oder diffuser Pankreasvergrößerung und weist typischerweise peripankreatische Entzündungszeichen im Sinne einer fokalen Imbibierung sowie kleine Mengen peripankreatischer Flüssigkeit auf.

\section{Necrotizing acute pancreatitis}

Die nekrotisierende Pankreatitis, die etwa 5-10\% der Fälle der AP ausmacht, ist häufiger bei der mittelschweren akuten Pankreatitis bzw. bei der schweren akuten Pankreatitis anzutreffen. Die Nekrose kann entweder das Pankreasparenchym, das peripankreatische Gewebe oder beide Kompartimente gleichzeitig betreffen. Dabei tritt am häufigsten die Kombinationsform mit Nekrosen im Pankreasparenchym sowie peripankreatisch bei $75 \%$ der nekrotisieren- 
den Pankreatitis auf. In diesem Fall zeigt sich radiologisch eine nicht kontrastmittelaufnehmende, heterogene, peripankreatische Ansammlung (collection), die typischerweise in der Bursa omentalis und im vorderen Pararenalraum angrenzend an die Gerota-Faszie zu finden ist. Eine parakolische Ausdehnung bis ins Becken sowie die subperitoneale Ausdehnung ins Mesenterium sind möglich.

In $20 \%$ der Fälle tritt lediglich eine peripankreatische Nekrose auf, in der das Pankreas regulär Kontrastmittel aufnimmt, jedoch im peripankreatischen Gewebe nekrotische Areale mit Ansammlungen (collection) zu finden sind, die Flüssigkeit und solide bzw. Fettkomponenten enthalten können. Am wenigsten häufig tritt die reine Pankreasnekrose auf (etwa $5 \%$ der Fälle). Bei dieser Form fehlt die peripankreatische Ansammlung vollständig. Radiologisch ist es nicht zu empfehlen, innerhalb der ersten 72 Stunden eine Bildgebung durchzuführen. Einerseits kann gerade in der Frühphase das Pankreas ödematös und nach Kontrastmittelgabe teilweise mit verminderter Anreicherung erscheinen, die Nekrosen vortäuschen. Andererseits demarkieren sich Parenchymnekrosen sicher erst frühestens nach 72 Stunden. Damit besteht die Gefahr einer Fehlklassifikation.

\section{Definition von Komplikationen}

\section{Acute peripancreatic fluid collection (APFC)}

Dieser Terminus wird mit „akute peripankreatische Flüssigkeitsansammlung“ (APFC) im Deutschen übersetzt. Diese Komplikation entsteht innerhalb der ersten 4 Wochen als frühe Komplikation einer interstitiellen ödematösen akuten Pankreatitis. Insgesamt beschreibt er eine homogene Flüssigkeitsansammlung ohne Nachweis von Nekrosen und ohne definierte Abkapselung. Bei vollständig fehlenden Nekroseanteilen beschränkt sich die Flüssigkeitsausdehnung auf die retroperitonealen Kompartimente. Akute peripankreatische Flüssigkeitsansammlungen befinden sich immer peripankreatisch ( $\bullet$ Abb. 2a, b). Bei Nachweis ähnlicher Flüssigkeitsformationen innerhalb des Pankreasparenchyms handelt es sich per Definition um eine akute Nekrose (ANC) ( $\vee$ Abb. 1b) und kann damit nicht mehr einer interstitiell-ödematösen Pankreatitis entsprechen, sondern setzt eine Klassifikation als nekrotisierende Pankreatitis voraus. In der Regel werden akute peripankreatische Flüssigkeitsansammlungen im Laufe der Zeit selbstständig resorbiert und sollten, um ggf. die Infektion einer ansonsten sterilen Flüssigkeitsansammlung zu vermeiden, nicht drainiert oder punktiert werden. Im klinischen Alltag kann die Differenzierung einer akuten peripankreatischen Flüssigkeitsansammlung von einer akuten nekrotischen Flüssigkeitsansammlung manchmal Schwierigkeiten bereiten. In Zweifelsfällen sollte hier die Bildgebung nach 1-2 Wochen wiederholt werden, was häufig eine genauere Diagnose ermöglicht [4]. Sollte sich die akute peripankreatische Flüssigkeitsansammlung nach 4 Wochen nicht aufgelöst haben, organisiert sie sich in der Regel und entwickelt eine Kapsel, sodass sie als späte Komplikation als Pankreaspseudozyste in Erscheinung tritt (siehe dort).
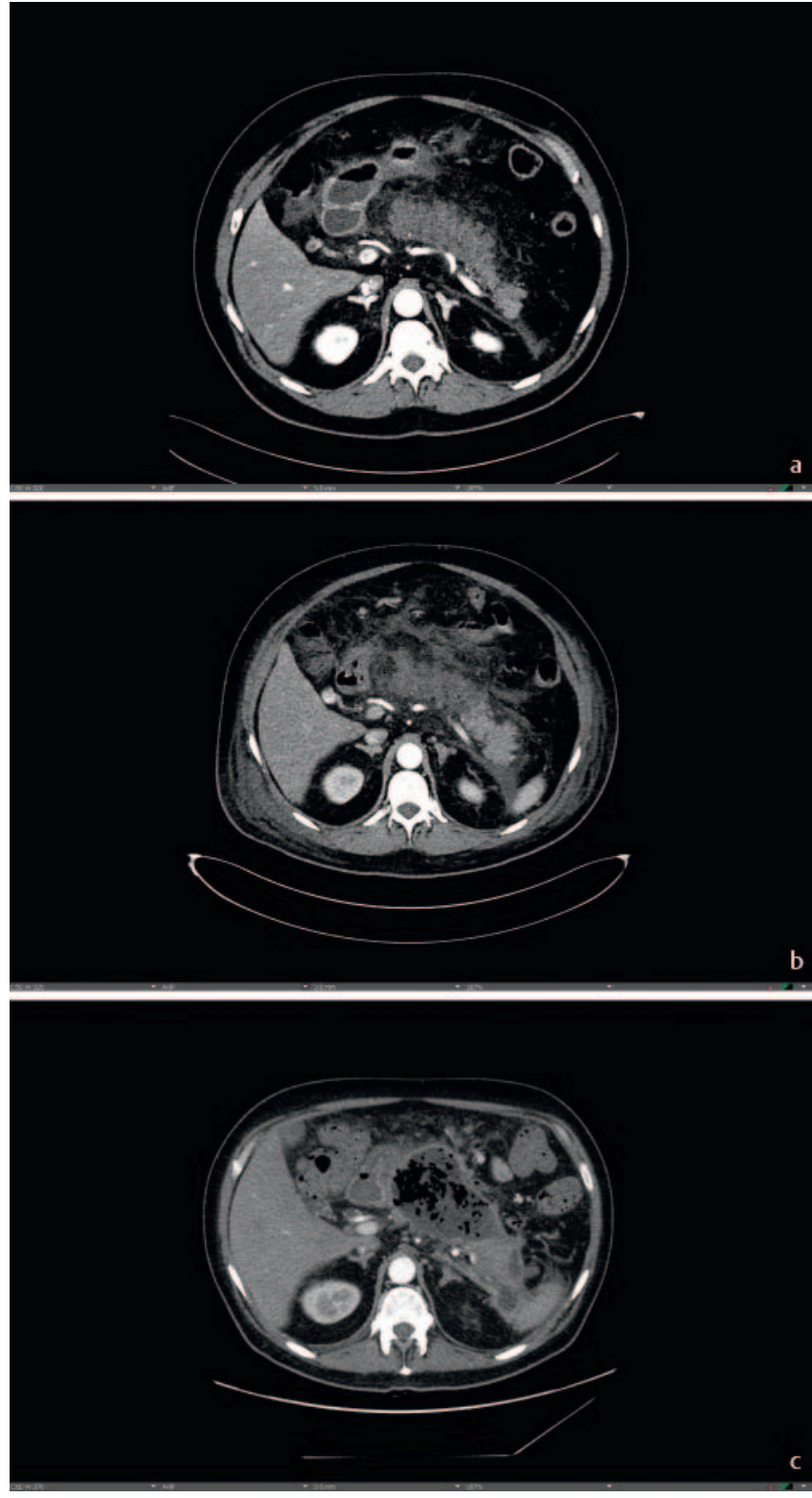

- Abb. 6 a Nekrotisierende Pankreatitis im Verlauf in der CT: In der Frühphase a mit bereits angedeuteter Minderperfusion im Pankreaskorpus; Verlauf nach 4 Tagen b mit Demarkierung der akuten Nekrose (ANC) im Korpus; Verlauf nach 8 Wochen c mit typischer später Komplikation und Ausbildung einer abgekapselten Nekrose (WON).

\section{Pancreatic pseudocyst}

Der Terminus wird mit „Pankreaspseudozyste“ übersetzt und stellt eine späte Komplikation einer interstitiell-ödematösen Pankreatitis dar. Definiert ist er durch eine abgekapselte Flüssigkeitsansammlung ohne relevante Nekroseanteile bei einer interstitiell-ödematösen Pankreatitis. Bezüglich der zeitlichen Definition entstehen Pseudozysten nach etwa 4 Wochen aus akuten peripankreatischen Flüssigkeitsansammlungen (APFC) und lediglich bei interstitiell-ödematösen Pankreatitiden (Abb. > 3a, b). Pseudozysten entwickeln sich in weniger als $10 \%$ bei einer interstitiell-ödematösen Pankreatitis [7]. Die Lokalisation von Pseudozysten ist in der Regel peripankreatisch ( $\triangleright$ Abb. 4$)$. 

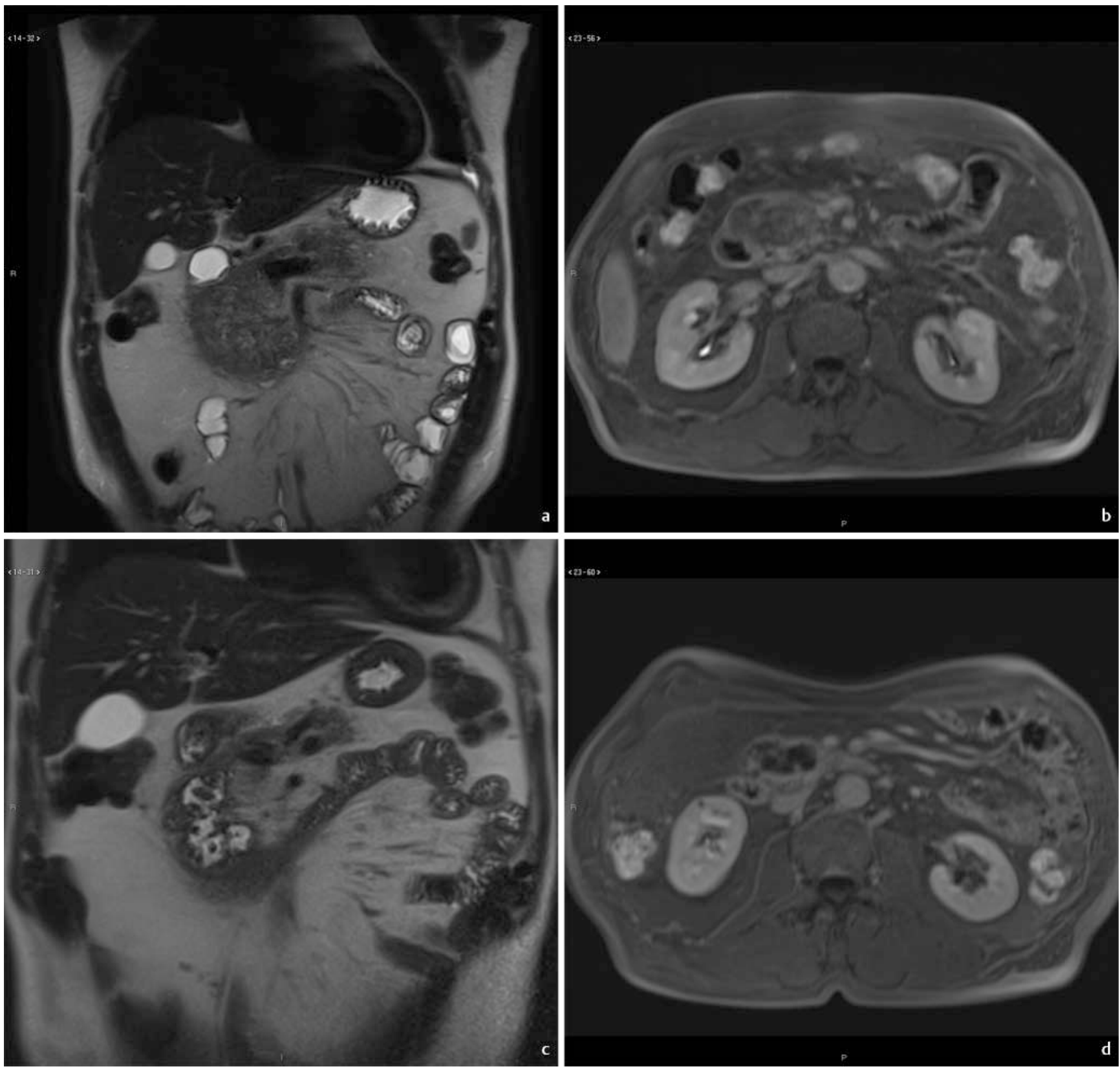

- Abb. 7 a Nekrotisierende Pankreatitis im Verlauf mit MRT: Beginnende Demarkierung der akuten Nekrose (ANC) im Pankreaskopf in T2w a und fettsaturierter T1w post KM b. Verlauf nach 9 Wochen: Als typische späte Komplikation zeigt sich eine abgekapselte Nekrose (WON) in T2w c und fettsaturierter T1w post KM d mit Fettanteilen und Zelldetritus.

\section{Acut necrotic collection (ANC)}

Dieser Begriff wird als „akute Nekrose“ übersetzt und stellt eine frühe Komplikation innerhalb der ersten 4 Wochen einer nekrotisierenden Pankreatitis dar. In diesem Fall lassen sich eine Flüssigkeitsansammlung sowie eine gleichzeitig vorliegende Nekrose ohne Nachweis einer Abkapselung in der Bildgebung nachweisen. Akute Nekrosen sind schlecht organisierte nekrotische Ansammlungen, die nur in einer nekrotisierenden Pankreatitis entstehen können ( $\vee$ Abb.5). Sie finden sich oft in der Bursa omentalis sowie in den pararenalen Kompartimenten und können sich in das Pankreas- parenchym als Nekrosen ausdehnen. Differenzialdiagnostisch unterscheiden sie sich von akuten peripankreatischen Flüssigkeitsansammlungen durch das Vorliegen von nekrotischem Gewebe, das als solide Anteile bzw. als fettige makroskopische Substrate innerhalb einer Flüssigkeitsansammlung erscheint. Im Rahmen der Konsentierung wurde im Expertengremium gesondert abgestimmt, ob die „ANC“ als „akute nekrotische Flüssigkeitsansammlung“ oder einfacher als „akute Nekrose“ bezeichnet werden sollte. Hier wurde mit starkem Konsens (> 75\%) für die Übersetzung „akute Nekrose“ entschieden. 


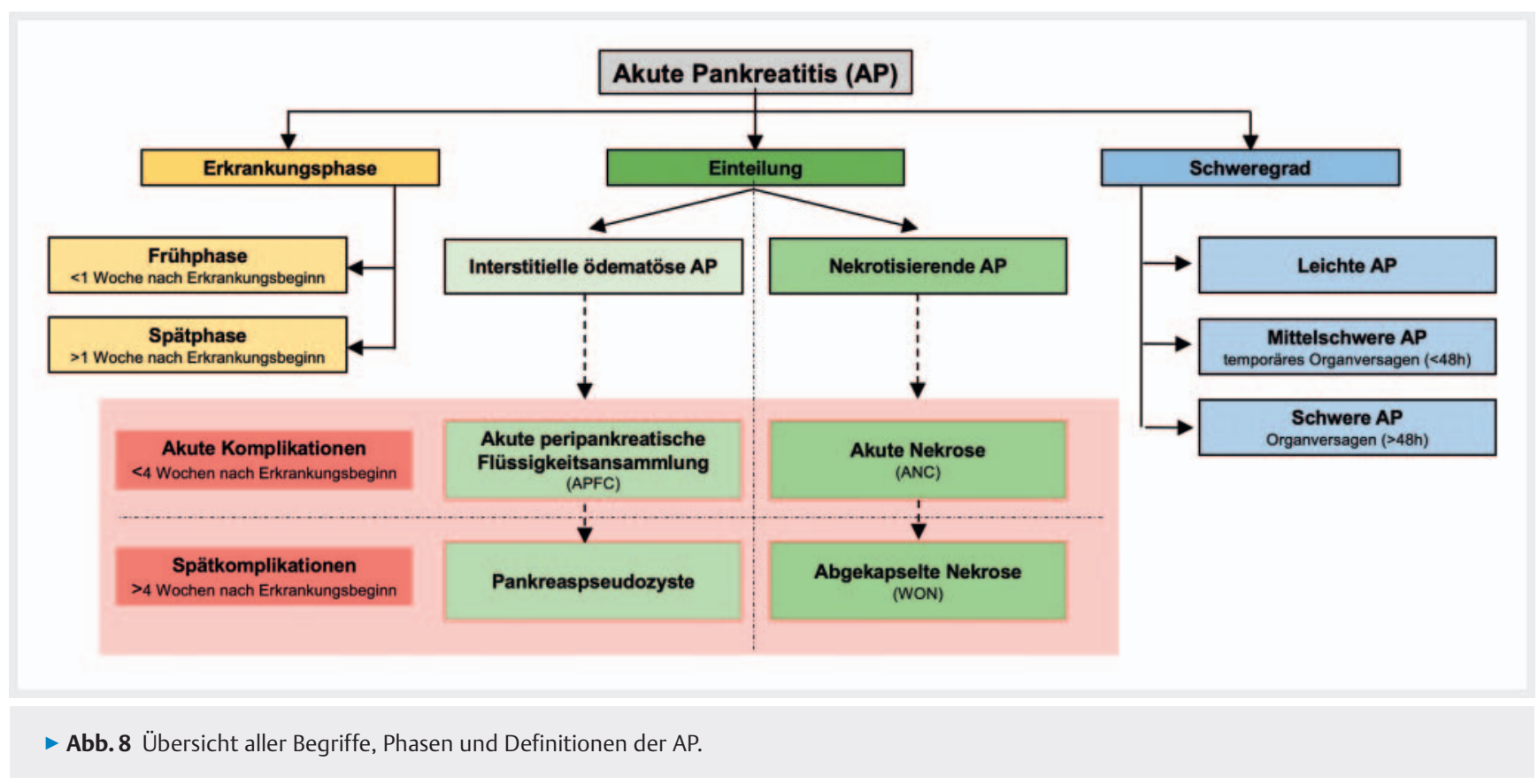

\section{Walled of necrosis (WON)}

Dieser Terminus wird als „abgekapselte Nekrose“ übersetzt und stellt eine späte Komplikation (4 Wochen nach Erkrankungsbeginn) bei Patienten mit einer nekrotisierenden Pankreatitis dar ( $\sim$ Abb.6a-c). Definiert ist die abgekapselte Nekrose durch eine abgekapselte Flüssigkeitsansammlung mit zusätzlich vorliegenden Nekroseanteilen, entweder des Pankreasparenchyms bzw. des peripankreatischen retroperitonealen Fettes oder von beiden Kompartimenten gleichzeitig. Diese abgekapselten Nekrosen mit „Zelldetritus“, Fettanteilen und Flüssigkeit sind oft mit der MRT sehr gut zu erfassen ( $\bullet$ Abb. 7a-d).

\section{Abstimmung im Rahmen der Konsensuskonferenz}

Die vorliegende Version der Übersetzung der revidierten AtlantaKlassifikation in die deutsche Sprache wurde gesondert im Rahmen der S3-Leitlinie am 12. Februar 2020 konsentiert und erreichte im Expertengremium eine Zustimmung von 97,3\%.

- Abb. 8 fasst dabei nochmal die wichtigsten verwendeten Begriffe und Definitionen der akuten Pankreatitis (AP) in einer Übersicht zusammen.

\section{Fazit}

Die Neuauflage der deutschen S3-Leitlinie zur Pankreatitis vereint erstmals alle Statements der akuten, chronischen und der Autoimmunpankreatitis in einer Leitlinie. Ziel des Expertengremiums war es darüber hinaus, dies auch in einer einheitlichen Sprachregelung zu dokumentieren. So wurden alle Begrifflichkeiten der revidierten Atlanta-Klassifikation zu einem deutschen Glossar ( Tab. 1) zusammengefasst, der zukünftig flächendeckend seine
Anwendung findet. Um bei den Abkürzungen der Komplikationen durch die Einführung neuer, deutscher Abkürzungen nicht zusätzliche Verwirrung zu stiften, einigte man sich darauf, bei Verwendung der deutschen Begrifflichkeiten in Klammern gesetzt die englischen Abkürzungen ergänzend zu verwenden. So soll künftig z. B. die „walled-off necrosis“ als „abgekapselte Nekrose (WON)“ oder die „acute necrotic collection“ als „akute Nekrose (ANC)“ im Deutschen verwendet werden.

Die Verwendung der deutschen Begriffe ermöglicht es zudem, sprachliche Unklarheiten und Übersetzungsfehler durch die vorherige „Eindeutschung“ jetzt klar zu definieren, wie z. B. „milde Pankreatitis“ korrekt als „leichte Pankreatitis“ zu beschreiben.

Die gesamte Leitlinienexpertengruppe ist sich dabei einig, dass auch für das Update künftiger deutscher Leitlinien eine solche einheitliche Sprachregelung richtungsweisend sein sollte.

Interessenkonflikt

Die Autorinnen/Autoren geben an, dass kein Interessenkonflikt besteht.

Literatur

[1] Bradley EL 3rd. A clinically based classification system for acute pancreatitis. Summary of the International Symposium on Acute Pancreatitis, Atlanta, Ga, September 11 through 13, 1992. Arch Surg 1993; 128: 586590. doi:10.1001/archsurg.1993.01420170122019

[2] Petrov MS, Windsor JA. Classification of the severity of acute pancreatitis: how many categories make sense? Am J Gastroenterol 2010; 105: 74-76. doi:10.1038/ajg.2009.597

[3] Foster BR, Jensen KK, Bakis G et al. Revised Atlanta Classification for Acute Pancreatitis: A Pictorial Essay. Radiographics 2016; 36: 675-687. doi:10.1148/rg.2016150097

[4] Banks PA, Bollen TL, Dervenis C et al. Classification of acute pancreatitis2012: revision of the Atlanta classification and definitions by international consensus. Gut 2013; 62: 102-111. doi:10.1136/gutjnl-2012-302779 
[5] Singh VK, Bollen TL, Wu BU et al. An assessment of the severity of interstitial pancreatitis. Clin Gastroenterol Hepatol 2011; 9: 1098-1103. doi:10.1016/j.cgh.2011.08.026

[6] Baker ME, Nelson RC, Rosen MP et al. ACR Appropriateness Criteria(R) acute pancreatitis. Ultrasound Q 2014; 30: 267-273. doi:10.1097| RUQ.0000000000000099
[7] Lenhart DK, Balthazar E]. MDCT of acute mild (nonnecrotizing) pancreatitis: abdominal complications and fate of fluid collections. Am J Roentgenol 2008; 190: 643-649. doi:10.2214/Am J Roentgenol.07.2761

[8] Layer P, Dellinger EP, Forsmark CE et al. Einteilung des Schweregrads der akuten Pankreatitis. Internationale multidisziplinäre klinische Klassifikation auf Basis pathogenetischer determinanten: Deutsche Version 2013. Z Gastroenterol 2013; 51: 544-550 